\title{
ANÁLISE MORFOLÓGICA DAS VIAS AÉREAS DE RATOS EXPOSTOS À FUMAÇA DO CIGARRO, E AO ÁLCOOL E SUA RELAÇÃO COM O EXERCÍCIO FÍSICO
}

Fabiola de Azevedo Mello ${ }^{1}$, Carolina Capelasso de Oliveira ${ }^{1}$, Gabriela Vidotto Cavalieri ${ }^{1}$, Armando Ribeiro Florido Neto $^{2}$, Lucas Agostini ${ }^{2}$, Ana Caroline Rippi Moreno ${ }^{2}$, Ana Karina Marques Salge ${ }^{3}$, Débora Tavares de Resende e Silva ${ }^{4}$, Patrícia Monteiro Seraphim ${ }^{2}$, Renata Calciolari Rossi ${ }^{1}$.

${ }^{1}$ Universidade do Oeste Paulista - UNOESTE, Presidente Prudente, SP; ${ }^{2}$ Faculdade de Ciências e Tecnologia de Presidente Prudente, SP; ${ }^{3}$ Universidade Federal de Goiás, Goiânia, GO; ${ }^{4}$ Universidade Federal Fronteira do Sul, Chapecó, SC. e-mail: renatacalciolari@terra.com.br

\section{RESUMO}

O objetivo do estudo foi quantificar e comparar, morfologicamente, o muco e o infiltrado inflamatório em ratos expostos à fumaça do cigarro, ao álcool e a ambos os agentes, incluindo a realização de exercício físico. Foram utilizados 40 ratos machos Wistar, divididos aleatoriamente em quatro grupos. Os animais controles realizaram exercícios físicos. Os outros animais foram expostos à fumaça do cigarro, a ingestão de álcool e ao treinamento físico, de acordo com o respectivo grupo. Foram coletados os pulmões dos animais para avaliar os achados. Não houve diferença significativa entre os grupos em relação à avaliação do perímetro da via aérea e a quantidade de células inflamatórias em vias aéreas dos animais. Conclui-se que não houve influência do exercício físico na produção de muco e infiltrado inflamatório em ratos expostos ao álcool e fumaça do cigarro. Desta forma, sugere-se a realização de novos estudos a fim de elucidar os resultados.

Palavras-chave: Tabagismo, transporte mucociliar, alcoolismo, exercício físico.

\section{Morphological analysis of the airways of rats exposed to cigarette smoke, and alcohol and their relationship with physical exercise}

\begin{abstract}
The aim of the study was to quantify and compare, morphologically, the mucus and inflammatory infiltrate in rats exposed to cigarette smoke, alcohol and both agents, including physical exercise. Forty male Wistar rats were used, randomly divided into four groups. Control animals performed physical exercises. The other animals were exposed to cigarette smoke, alcohol intake and physical training, according to the respective group. The lungs of the animals were collected to assess the findings. There was no significant difference between the groups regarding the assessment of the airway perimeter and the amount of inflammatory cells in the animals' airways. It was concluded that there was no influence of physical exercise on the production of mucus and inflammatory infiltrate in rats exposed to alcohol and cigarette smoke. Thus, it is suggested to carry out further studies in order to elucidate the results.
\end{abstract}

Keywords: Smoking, mucociliary transport, alcoholism, physical exercise.

\section{INTRODUÇÃO}

O tabagismo e o alcoolismo são considerados as principais e maiores dependências humanas que vêm sendo estudados há décadas, e a associação e correlação positiva entre eles já foi demonstrada anteriormente ${ }^{1,2}$.

Dentro da saúde pública, tanto o tabagismo quanto o alcoolismo, estão dentre os principais fatores de risco para doenças crônicas 
não-transmissíveis, ocasionado milhões de mortes anualmente ${ }^{3}$.

O alcoolismo vem comumente associado a fatores como aumento de desemprego, doenças hepáticas, acidentes de trânsito ${ }^{4}$ e transtornos psiquiátricos, como a depressão ${ }^{5}$. Já o tabagismo, é mais relacionado a doenças respiratórias como enfisema pulmonar, bronquite, infecções e tuberculose, além de aumentar o risco de câncer, principalmente o de pulmão ${ }^{6}$.

Apesar de ser pouco documentado, o consumo excessivo de álcool também pode estar associado a alterações do sistema respiratório como insuficiência respiratória, edema pulmonar e aumento da susceptibilidade a infecções do trato respiratório ${ }^{7,8}$.

Uma das formas de reduzir os danos ao sistema respiratório e melhorar a função pulmonar é por meio da prática de atividades físicas. A prática de exercício físico regular atua de forma a reduzir os efeitos maléficos por meio de um mecanismo anti-inflamatório e antioxidante. No caso do tabagismo, o exercício físico e o uso do tabaco interagem de forma antagônica: marcadores inflamatórios que são produzidos no pulmão e estão relacionados ao declínio cardiorrespiratório são suprimidos pela realização do exercício físico ${ }^{9}$.

A atividade física de moderada a alta intensidade já demonstrou contribuição na redução do declínio da função pulmonar em fumantes ${ }^{9}$ e também proteção contra os efeitos lesivos do álcool sobre lesões de isquemia e reperfusão, por meio de aumento da atividade de sistemas antioxidantes em pulmão de ratos sedentários e treinados ${ }^{10}$.

As doenças respiratórias são o segundo principal motivo de internações hospitalares no Brasil e a principal causa de morte durante internações, sendo responsável por 19,5\% dos casos, o que gera altos custos de saúde para o país $^{11}$. Desta forma, é imprescindível definir métodos que possam reduzir os efeitos maléficos da saúde do sistema respiratório a fim de traçar estratégias de saúde pública para contribuir na redução dos números de internações e mortes.

Sendo assim, o objetivo do presente estudo foi quantificar e comparar, morfologicamente, o muco e o infiltrado inflamatório em pulmões de ratos praticantes de exercício físico, expostos à fumaça do cigarro, ao álcool e a ambos os agentes.

\section{METODOLOGIA}

O projeto foi executado após a aprovação do Comitê de Ética em Animais da Universidade do Oeste Paulista (protocolo 2695). Os procedimentos relacionados aos protocolos de experimentação (expostos à fumaça do cigarro e ao álcool) até a eutanásia dos animais foram executados na Faculdade de Ciências e Tecnologia de Presidente Prudente e aprovado sob o processo no 1/2012.

O procedimento relacionado à realização de técnicas histológicas foi realizado no laboratório de anatomia patológica da Universidade do Oeste Paulista (UNOESTE).

Procedimento experimental

Junto ao biotério experimental, foram obtidos 40 ratos Wistar, machos, pesando em torno de 200 gramas e permaneceram no biotério para animais da Faculdade de Ciências e Tecnologia de Presidente Prudente, em gaiolas plásticas coletivas $(30 \times 16 \times 19 \mathrm{~cm})$ com 5 animais por gaiola, sob temperatura média de $22 \pm 2$ e ciclo claro/escuro de $12 / 12 \mathrm{~h}$, sendo o ciclo claro iniciado as 7:00 horas.

Os animais foram divididos em quatro grupos e todos os grupos foram compostos por 10 animais/cada:

- GCE: Grupo controle exercício, não foram expostos à fumaça de cigarro, não ingeriram álcool e praticaram exercícios físicos;

- GTE: Grupo tabagista e exercício, expostos à fumaça de cigarro e praticaram exercícios físicos;

- GAE: Grupo alcoólico e exercício, ingeriram álcool e praticaram exercícios físicos;

- GTAE: Grupo tabagista, alcoólico e exercício, foram expostos à fumaça de cigarro, ingeriram álcool e praticaram exercícios físicos.

Protocolo de exposição à fumaça

Os animais que foram expostos à fumaça do cigarro passaram por uma fase de adaptação que durou 5 dias e correspondeu a 2 cigarros por 10 min uma vez ao dia. A fase experimental durou 60 dias e correspondeu a 4 cigarros por 30 min duas vezes ao dia durante 5 dias na semana, ou seja, a combustão de 8 cigarros por dia. Esta foi a dose suportada segundo Paiva et al. ${ }^{12}$ e Castardeli et al. ${ }^{13}$.

Foi utilizada uma caixa com estrutura de alumínio e vidro $(100 \times 44 \times 44 \mathrm{~cm})$, hermeticamente fechada e dividida em dois compartimentos por um tabique de vidro escuro com cinco pertuitos de uma polegada de 
diâmetro para a câmara de inalação. Os cigarros acesos ficaram em um dos compartimentos apoiados em um suporte e no outro compartimento ficaram 5 ratos expostos à fumaça dentro de uma gaiola. $O$ experimento aproximou-se de um fumo passivo, onde os animais apenas foram expostos à fumaça através de orifícios entre as duas câmaras. Isso ocorreu porque a câmara de queima de cigarros foi conectada a uma fonte de ar comprimido com um fluxo de $10 \mathrm{l} / \mathrm{min}$ que permitiu a combustão dos cigarros e a condução da fumaça para a câmara de exposição na qual foi feita a exaustão da mistura.

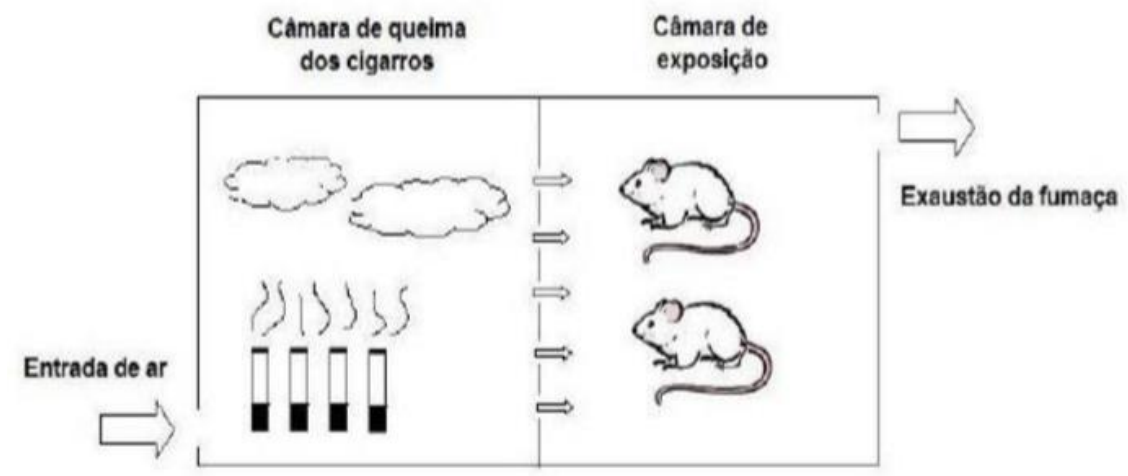

Figura 1. Desenho esquemático da câmara de inalação utilizada para exposição dos animais à fumaça.

Protocolo de ingestão de álcool

Os ratos que ingeriram etanol passaram por um processo de adaptação. A concentração de etanol aumentou de $5 \%$ v/v no 10 e 20 dia, $7,5 \% \mathrm{v} / \mathrm{v}$ no 3 ㅇ e 4 을 dia, até chegar à concentração experimental de $10 \% \mathrm{v} / \mathrm{v}$ que permaneceu assim por 60 dias.

\section{Protocolo de treinamento físico}

As sessões de treinamento foram realizadas cinco vezes por semana, durante sessenta dias, sendo a primeira semana, de adaptação. Na semana de adaptação os animais realizaram sessões de treinamento com duração de 15, 30, 45, $60 \mathrm{~min}$. Nas semanas seguintes, os animais foram submetidos a sessões de treinamento de $60 \mathrm{~min}$.

O treinamento foi feito em esteira rolante, própria para animais de pequeno porte, construída pelo Serviço Nacional de Aprendizagem Industrial (SENAI) de Presidente Prudente $^{14}$.

A esteira foi composta por dois roletes com distâncias definidas entre si, onde por eles deslizaram em uma manta de algodão impermeável que provocou o movimento dos animais em estudo. Os eixos foram acionados por um motor de 12 volts com rotação variável para atribuir uma velocidade ajustável entre 0 a $10 \mathrm{~m} / \mathrm{min}$. Foram elaboradas oito pistas, sendo cada uma com $100 \mathrm{~mm}$ de largura por $600 \mathrm{~mm}$ de comprimento, independentes uma das outras. A metade anterior de cada uma das pistas com suas laterais foram pintadas de preto e cobertas por madeira a fim de que essa região estivesse completamente escura. Essa medida foi necessária, pois os animais tenderam a fugir, procurando locais escuros ${ }^{15}$.

\section{Coleta do material}

Os animais foram eutanasiados por meio dos anestésicos Quetamina e Xilazina $(40 \mathrm{mg} / \mathrm{kg}$ do peso corporal) por via intraperitoneal. Posteriormente, foi realizada autópsia dos animais para retirada dos pulmões para análise. Uma cânula plástica foi introduzida e em seguida, foi instilada formalina tamponada $10 \%$ na região da traquéia para que a estrutura morfológica dos pulmões e a pressão constante de $20 \mathrm{cmH}_{2} \mathrm{O}$ se mantivessem inalteradas ${ }^{16}$.

\section{Desidratação e inclusão do material}

$$
\text { Realizou-se uma desidratação }
$$

homogênea dos tecidos através de imersão numa bateria de soluções alcoólicas em concentrações graduais para evitar danos na estrutura tecidual. Para a inclusão do material, o mesmo foi imerso em parafina líquida a $60^{\circ} \mathrm{C}$, obtendo-se, em temperatura ambiente, um bloco que continha o fragmento de tecido em seu interior. Posteriormente, foram submetidos à microtomia, onde os cortes coletados de $5 \mu \mathrm{m}$ foram 
colocados em lâminas de vidro e encaminhados para a coloração.

Coloração para visualização do infiltrado inflamatório

Para a visualização das células inflamatórias foi utilizado o método de coloração Hematoxilina e Eosina. O protocolo desta técnica consistiu inicialmente em desparafinizar, alcoolizar (álcool absoluto) e hidratar. Em seguida, mergulhou-se em Hematoxilina de Harris por 5 min e lavou-se em água corrente, depois a lâmina foi mergulhada no diferenciador por $4 \mathrm{seg}$ (álcool ácido clorídrico) e lavada rapidamente em água corrente. Após esta fase, o fragmento foi mergulhado em Eosina por 5 min, seguido por álcool absoluto, para posterior montagem em meio sintético com entelan.

\section{Coloração para análise da presença de muco}

Para quantificar a presença de muco no parênquima pulmonar foi feita a coloração de Alcian Blue que consiste em desparafinização e hidratação em água destilada. Após este procedimento, as lâminas foram mergulhadas em Alcian Blue por 30 min (filtrar) e lavadas em água corrente por $10 \mathrm{~min}$. Posteriormente, utilizou-se ácido periódico $0,5 \%$ por 15 min e água corrente por $10 \mathrm{~min}$, esperou-se secar. Reativo de Schiff 15 min, metabissulfato de sódio $1 \%$ por 5 min, água corrente $10 \mathrm{~min}$, desidratação e montagem em meio sintético com entelan.

Análise de resultados

Depois de realizada a coloração de hematoxilina eosina, as células foram quantificadas na região peribrônquica e classificadas em leve, moderado e acentuado de acordo com Mauad ${ }^{17}$. A quantificação de muco foi realizada através do sistema analisador de imagens Image Pro Plus ${ }^{\circ}$ para posterior análise e os resultados foram expressos em $\mu \mathrm{m}^{2}$.

Para a análise dos resultados foi confeccionado um banco de dados eletrônico. Como se trata de amostras independentes utilizou-se o teste de ANOVA nos casos de distribuição paramétrica e Kruskall-Wallis nos casos de distribuição não paramétrica. Foram significativos os valores de $p<0,05$.

\section{RESULTADOS}

Este estudo avaliou as vias aéreas contidas em pulmões de 40 ratos expostos à fumaça do cigarro, ao álcool e a ambos os agentes simultaneamente, incluindo a realização de exercício físico. Não houve diferença significativa entre os grupos em relação à avaliação do perímetro da via aérea $(p=0,489)$ e a quantidade de células inflamatórias em vias aéreas dos animais $(p=0,207)$ (Tabela 1 e 2).

Tabela 1. Avaliação do perímetro da via aérea de modelos experimentais praticantes de exercício físico expostos ao tabaco e álcool.

\begin{tabular}{lcc}
\hline Grupos & $\mathrm{n}(\%)$ & $\mathrm{X} \pm \mathrm{DP}\left(10^{4}\right)$ \\
\hline GCE & $10(25)$ & $387,4 \pm 238,8$ \\
\hline GAE & $10(25)$ & $275,4 \pm 111,8$ \\
\hline GTAE & $10(25)$ & $381,6 \pm 144,3$ \\
\hline GTE & $10(25)$ & $381,7 \pm 193,9$ \\
\hline Total & $40(100)$ &
\end{tabular}

$p=0,489$. Grupos: GCE: Controle e Exercício; GAE: Alcoólico e Exercício; GTAE: Tabagista, Alcoólico e Exercício; GTE: Tabagista e Exercício.

Tabela 2. Quantificação de células inflamatórias em vias aéreas de modelos experimentais praticantes de exercício físico expostos ao tabaco e álcool.

\begin{tabular}{lcc}
\hline Grupos & $\mathrm{n}(\%)$ & $\mathrm{X} \pm \mathrm{DP}$ \\
\hline GCE & $10(25)$ & $52,3 \pm 27,7$ \\
\hline GAE & $10(25)$ & $11,7 \pm 3,9$ \\
\hline GTAE & $10(25)$ & $26,7 \pm 8,4$ \\
\hline GTE & $10(25)$ & $36,5 \pm 13,8$ \\
\hline Total & $40(100)$ &
\end{tabular}

$p=0,207$. Grupos: GCE: Controle e Exercício; GAE: Alcoólico e Exercício; GTAE: Tabagista, Alcoólico e Exercício; GTE: Tabagista e Exercício.

\section{DISCUSSÃO}

Em nossa pesquisa não foi observada nenhuma diferença significante em relação a prática de exercícios em ratos expostos ao álcool, ao tabaco e ambos, diferente de outro trabalho que referiu melhora da inflamação do tecido pulmonar por meio da prática de exercícios físicos ${ }^{9}$.

A exposição crônica ao tabagismo gera alteração do transporte mucociliar e assim, aumento da capacidade de adesão de bactérias às células epiteliais, de forma que há uma elevada reação inflamatória ${ }^{18}$. Entretanto, essas alterações não duram apenas enquanto há a prática do tabagismo, pois autores afirmam que o processo inflamatório da mucosa respiratória provocado pelo tabaco continua mesmo após um período de sete meses de recuperação ${ }^{19}$, ou seja, mesmo após a cessação do hábito a inflamação permanece por um período de tempo, o que 
poderia justificar parte dos achados do presente estudo, para o grupo exposto ao tabaco, visto que não avaliamos o efeito do exercício após o abandono do tabagismo.

Sabe-se que indivíduos ativos fisicamente possuem menores chances de serem acometidos por efeitos negativos de citocinas próinflamatórias, visto que a atividade física reduz os níveis dessas citocinas. Entretanto, há teorias de que o excesso dessas atividades pode levar a um maior estresse oxidativo e aumento da inflamação tecidual ${ }^{20}$.

Para as lesões pulmonares geradas devido a exposição ao álcool, já foi demonstrado que o exercício físico, mesmo sendo de baixa intensidade, não demonstrou efeitos positivos em relação a inflamação dos pulmões ${ }^{21}$, corroborando com a presente pesquisa. Pesquisadores observaram que ratos que ingeriram álcool e realizaram atividade física apresentaram áreas mais extensas de edema e infiltrado inflamatório comparado aos ratos que ingeriram álcool e eram sedentários ${ }^{21}$. Embora não tenhamos encontrado quantidade menores de infiltrado inflamatório e muco nos pulmões de ratos expostos ao álcool e atividade física, neste estudo não comparamos com ratos sedentários para avaliar a possibilidade de piora da inflamação.

Já é do conhecimento da comunidade científica que o exercício físico regular contribui na manutenção da função e da força muscular além de melhorar a atividade cardiorrespiratória e qualidade de vida. Porém, vale ressaltar que a intensidade e o tempo de atividade física influenciam diretamente na resposta do corpo ${ }^{22}$. Alguns autores evidenciaram que o exercício físico intenso pode estar relacionado com o aumento da incidência de risco para infecções do sistema respiratório ${ }^{23}$. Sabendo disso, a intensidade da atividade física realizada pelos animais na presente pesquisa, pode ter influenciado a não encontrar resultados significantes.

Uma pesquisa que avaliou a inflamação pulmonar em ratos sedentários e praticantes de atividades físicas expostos ao álcool encontrou edema pulmonar e extenso infiltrado inflamatório nos ratos expostos ao álcool ${ }^{21}$. Nesta mesma pesquisa os autores não observaram diferença entre os grupos controles sedentário e treinados em relação a diferenças morfológicas, mas observaram aumento do edema pulmonar e do número de macrófagos pigmentados do grupo exposto álcool e treinado comparado ao grupo exposto álcool controle ${ }^{21}$. O que diferiu do nosso estudo foi que a exposição ao álcool dos autores anteriores durou 120 dias e o exercício, que era natação, era realizado apenas 20 minutos por dia. Já na presente pesquisa, a exposição ao álcool durou 60 dias e os exercícios eram realizados por 60 minutos por dia, portanto, a quantidade de dias de ingestão de álcool pode ter sido o principal motivo entre a diversidade de resultados entre os estudo, demonstrando que além a intensidade do exercício, o tempo de exposição ao álcool também influencia no surgimento das inflamações.

\section{CONCLUSÃO}

Conclui-se que não houve influência do exercício físico na produção de muco e infiltrado inflamatório em ratos expostos ao álcool e fumaça do cigarro. Desta forma, sugere-se a realização de novos estudos a fim de elucidar melhor os resultados.

\section{DECLARAÇÃO DE CONFLITO DE INTERESSE}

Os autores declaram não haver qualquer potencial conflito de interesse que possa interferir na imparcialidade deste trabalho científico.

\section{REFERÊNCIAS}

1. Burns DM. Epidemiology of smoking-induced cardiovascular disease. Prog Cardiovasc Dis. 2003;46(1):11-29.

https://doi.org/10.1016/\$0033-0620(03)00079-3

2. Glass JM, Adams KM, Nigg JT, Wong MM, Puttler LI, Buu A, et al. Smoking is associated with neurocognitive deficits in alcoholism. Drug Alcohol Depend. 2006;82(2):119-26. https://doi.org/10.1016/j.drugalcdep.2005.08.01 $\underline{3}$

3. World Health Organization. WHO report on the global tobacco epidemic, 2011. Warning about the dangers of tobacco. Geneve: World Health Organization; 2011. https://doi.org/10.15288/jsas.2002.s14.91

4. Perkins HW. Surveyingthedamage: a reviewofresearchonconsequencesofalcoholmisus e in collegepopulations. J Stud Alcohol. 2002;(Suppl 14):91-100. 
5. Ramstedt M. Alcoholand suicide in 14 European countries. Addiction. 2001;96 (Suppl 1):S59-75. https://doi.org/10.1080/09652140020021189

6. World Health Organization. Tobacco health warnings. World no tobacco day. Geneve: World Health Organization; 2009.

7. Burnham EL, Halkar R, Burks M, Moss M. The effects of alcohol abuse on pulmonary alveolarcapillary barrier function in humans. Alcohol. 2009;44(1):8-12.

https://doi.org/10.1093/alcalc/agn051

8. Fiske $C T$, Hamilton $C D$, Stout JE. Alcohol use and clinical manifestations of tuberculosis. J Infect. 2009;58(5):395401. https://doi.org/10.1016/i.jinf.2009.02.015

9. Garcia-Aymerich J, Lange P, Benet M, Schnohr P, Antó JM. Regular physical activity modifies smoking-related lung function decline and reduces risk of chronic obstructive pulmonary disease: a population-based cohort study. Am J Respir Crit Care Med. 2007;175(5):458-63. https://doi.org/10.1164/rccm.200607-8960C

10. Mussi RK, Camargo EA, Ferreira T, De Moraes $C$, Delbin MA, Toro IF, et al. Exercise training reduces pulmonar ischaemia-reperfusion-induced inflammatory responses. Eur Respir J. 2008;31:645-

\section{9. https://doi.org/10.1183/09031936.00015607}

11. Gomes HG, Dias SM, Gomes MS, Medeiros JSN, Ferraz LP, Pontes FL. Perfil das internações hospitalares no Brasil no período de 2013 a 2017. Rev Interd. 2017;10(4):96-104. Available from: https://revistainterdisciplinar.uninovafapi.edu.br index.php/revinter/article/view/1322.

12. Paiva SAR, Zornoff LAM, Okoshi MP, Okoshi K, Cicogna AC, Campana AO. Behavior of cardiac variables in animals exposed to cigarette smoke. Arq Bras Cardiol. 2003;81(3):225-228. Available from:

https://www.scielo.br/scielo.php?script=sci artte xt\&pid=S0066-782X2003001100002.

https://doi.org/10.1590/S0066-

$\underline{782 \times 2003001100002}$

13. Castardeli E, Paiva RAE, Matsubara BB, Matsubara LS, Minicucci MF, Azevedo PS, et al. A exposição crônica à fumaça de cigarro resulta em remodelação cardíaca e função ventricular comprometida em ratos. Arq Bras Cardiol. 2005;84:320-4. Available from: https://www.scielo.br/scielo.php?pid=S0066782X2005000400009\&script=sci arttext. https://doi.org/10.1590/S0066$\underline{782 \times 2005000400009}$

14. Andrew RJ. Treadmill for small laboratory animals. J Appl Physiol. 1965;20(3):5724. https://doi.org/10.1152/jappl.1965.20.3.572

15. Camargo Filho JCS, Vanderlei LCM, Camargo $R C T$, Oliveira DAR, Oliveira Júnior AS, Dal Pai V, et al. Análise histológica, histoquímica e morfométrica do músculo sóleo de ratos submetidos a treinamento físico em esteira rolante. Arq Ciênc Saúde. 2005;12:196-5.

16. Toledo AC, Magalhaes RM, Hizume DC, et al. Aerobic exercise attenuates pulmonary injury induced by chronic exposure to cigarette smoke in mice. Eur Respir J. 2012;39(2):254-64. https://doi.org/10.1183/09031936.00003411

17. Van Rensen ELJ, Evertse CE, Van Schadewijk WAAM, Van Wijngaarden S, Ayre, G, Mauad, T, et al. Eosinophils in bronchial mucosa of asthmatics after allergen challenge: effect of anti-IgE treatment. Allergy. 2009;64(1):7280. https://doi.org/10.1111/i.13989995.2008.01881.x

18. Tamashiro E, Cohen NA, Palmer JN, Lima WTA. Efeitos do cigarro sobre o epitélio respiratório e sua participação na rinossinusite crônica. Braz J Otorhinolaryngol. 2009;75(6):9037. 86942009000600022 https://doi.org/10.1590/S1808-

19. Hamm JT, Yee S, Rajendran N, Morrissey RL, Richter SJ, Misra M. Histologicalalterations in male $\quad \mathrm{A} / \mathrm{J} \quad$ micefollowingnoseonlyexposuretotobaccosmoke. InhalationToxicol. 2007 May;19(5):405-18. https://doi.org/10.1080/08958370601174875

20. Goel R, Majeed F, Vogel R, Corretti MC, Weir $M$, Mangano $C$, et al. Exercise-induced hypertension, endothelial dysfunction, and coronary artery disease in a marathon runner. Am J Cardiol. 2007;99(5):743-4. https://doi.org/10.1016/i.amjcard.2006.09.127 
21. Xavier AM, Goessler KF, Ferrari O, Juliani LC, De Andrade FG, Ramos SP. Efeito do treinamento físico no pulmão de ratos submetidos à ingestão alcoólica. Rev Bras Med Esporte. 2010;16(6):43640. https://doi.org/10.1590/S1517-

86922010000600008

22. Leeuwen PBVW, Arets HGM, Van der Ente CK, Beekman JM. Infection, inflammation and exercise in cystic fibrosis. Respir Res. 2013;14(1):14-32. https://doi.org/10.1186/14659921-14-32

23. Kurowski M, Jurczyk J, Jarzebska M, Moskwa $\mathrm{S}$, Malowska JS, Krystifuak $\mathrm{H}$, et al. Association of serum Clara cell protein $\mathrm{CC} 16$ with respiratory infections and immune response to respiratory pathogens in elite athletes. Respir Res. 2014;15(1):45. https://doi.org/10.1186/14659921-15-45 